Published in final edited form as:

Angew Chem Int Ed Engl. 2014 March 3; 53(10): 2648-2653. doi:10.1002/anie.201309718.

\title{
Copolymerization of Metal Nanoparticles: A Route to Colloidal Plasmonic Copolymers*
}

\author{
Prof. Kun Liu'[+], \\ State Key Laboratory of Supramolecular Structure and Materials, College of Chemistry, Jilin \\ University, Changchun 130012 (P. R. China). Department of Chemistry, University of Toronto, 80 \\ Saint George street, Toronto M5S 3H6, Ontario (Canada) \\ Ariella Lukach[+], \\ Department of Chemistry, University of Toronto, 80 Saint George street, Toronto M5S 3H6, \\ Ontario (Canada)
}

\section{Kota Sugikawa,}

Department of Chemistry, University of Toronto, 80 Saint George street, Toronto M5S 3H6, Ontario (Canada)

\section{Siyon Chung,}

Department of Chemistry, University of Toronto, 80 Saint George street, Toronto M5S 3H6, Ontario (Canada)

\section{Dr. Jemma Vickery,}

Department of Chemistry, University of Toronto, 80 Saint George street, Toronto M5S 3H6, Ontario (Canada)

\section{Dr. Heloise Therien-Aubin,}

Department of Chemistry, University of Toronto, 80 Saint George street, Toronto M5S 3H6, Ontario (Canada)

\section{Prof. Bai Yang,}

State Key Laboratory of Supramolecular Structure and Materials, College of Chemistry, Jilin University, Changchun 130012 (P. R. China)

Prof. Michael Rubinstein, and

Department of Chemistry, University of North Carolina, Chapel Hill, NC 27599-3290, USA

\section{Prof. Eugenia Kumacheva}

Department of Chemistry, University of Toronto, 80 Saint George street, Toronto M5S 3H6, Ontario (Canada)

Kun Liu: kliu@jlu.edu.cn; Eugenia Kumacheva: ekumache@chem.utoronto.ca

\footnotetext{
**E.K. thanks Canada Research Chair support (NSERC Canada). K.L. thanks the Ontario Ministry of Economic Development and Innovation for the Post-Doctoral Fellow award. Assistance of Prof. N. Coombs and Ilya Gourevich is appreciated.

Correspondence to: Kun Liu, kliu@jlu.edu.cn; Eugenia Kumacheva, ekumache@chem. utoronto.ca.

${ }^{[+]}$These authors contributed equally to this work.

Supporting information for this article is available on the WWW under http://www.angewandte.org or from the author.
} 


\section{Abstract}

The resemblance between colloidal and molecular polymerization reactions has been recognized as a powerful tool for the fundamental studies of polymerization reactions, as well as a platform for the development of new nanoscale systems with desired properties. Future applications of colloidal polymers will require nanoparticle (NP) ensembles with a high degree of complexity that can be realized by hetero-assembly of NPs with different dimensions, shapes and compositions. In the present work, we have developed a method to apply strategies from molecular copolymerization to the co-assembly of gold nanorods with different dimensions into random and block copolymer structures (plasmonic copolymers). The approach was extended to the coassembly of random copolymers of gold and palladium nanorods. A kinetic model validated and further expanded the kinetic theories developed for molecular copolymerization reactions.

\section{Keywords}

Gold Nanorods; Plasmonic Polymers; Self-Assembly; Step-Growth Copolymerization; Colloids

Self-assembled one-dimensional nanostructures are rapidly gaining attention due to to their fundamental importance as model systems for molecular polymerization reactions ${ }^{[1]}$ and potential technological applications. ${ }^{[2]}$ In particular, linear polymer-like structures assembled from metal nanoparticles (NPs) (plasmonic polymers ${ }^{[3]}$ ) exhibit strong interactions between the surface plasmon resonances of NP repeat units, which lead to promising applications of plasmonic polymers in nanocircuits, ${ }^{[4]}$ nanoscale light transport, ${ }^{[5]}$ nanoantennae, ${ }^{[6]}$ and sensing. ${ }^{[2 b, 7]}$ The optical properties of plasmonic polymers can be controlled and tuned by changing their degree of polymerization, NP orientation and interparticle spacing. ${ }^{[8]}$

The next step in developing new plasmonic nanostructures is to move from homopolymer to copolymer structure by assembling NPs with different sizes ${ }^{[9]}$ and compositions, ${ }^{[10]}$ and by varying the order, in which distinct NPs are incorporated in the chain. Similar to the selfassembly of colloidal homopolymers, ${ }^{[11]}$ the self-assembly of plasmonic copolymers can greatly benefit from synthetic strategies developed for molecular copolymerization, ${ }^{[12]}$ if a proper use of analogies and differences between these two processes is made.

Currently, linear co-assembly of distinct types of NPs is limited to few examples of alternating or block copolymers formed by spherical NPs. ${ }^{[13]}$ No quantitative analysis of the co-assembly process and its comparison to copolymerization reactions has been reported. Linear co-assembly of anisotropic NPs, e.g., nanorods (NRs) with different dimensions and compositions has not been studied, although such structures may have new optical properties, due to higher-order plasmonic modes. ${ }^{[9,14]}$

In the present work, we report a molecular copolymerization approach to the co-assembly of gold NRs with different dimensions into random and block copolymer structures. For each structure we analyzed the change in composition, degree of polymerization, and heterogeneity. The approach is further extended to the copolymerization of NRs with different compositions. The proposed methodology offers a polymer synthesis-based 
strategy for producing one-dimensional nanomaterials with varying compositions and structures. This work is also important for fundamental studies of optical properties of linear plasmonic heterostructures. In particular, the theoretical prediction of new Fano plasmon modes in chains composed of gold NRs with different dimensions can be experimentally examined by single particle spectroscopy. ${ }^{[15]}$

To examine copolymerization of NRs with different lengths, we synthesized gold NRs with the mean diameter and length of 12 and $99 \mathrm{~nm}$, respectively, ${ }^{[16]}$ and fine-tuned the NR length in an end-etching process using $\mathrm{HCl}$ and $\mathrm{H}_{2} \mathrm{O}_{2}{ }^{[17]}$. With increasing etching time, the longitudinal surface plasmon resonance (LSPR) band ${ }^{[18]}$ of the NRs gradually blue-shifted, while the transverse surface plasmon band was invariant (Figure 1a), indicating the reduction NR length and a close-to-constant diameter of NRs. After a particular time, the etching process was terminated, yielding NRs with a desired length. Figure $1 \mathrm{~b}-\mathrm{d}$ show scanning transmission electron microscopy (STEM) images of the NRs with different lengths used in the present work. The resultant NR were end-functionalized with thiolterminated polystyrene (PS) molecules with the molecular weight $M_{\mathrm{n}}=12000 \mathrm{~g} \mathrm{~mol}^{-1}$. [11c]

The end-to-end association of NRs in chains was initiated by adding water to the solution of PS-functionalized NRs in dimethylformamide (DMF), to a final water concentration $C_{\mathrm{w}}=15$ $\mathrm{wt} \%$. To minimize the surface energy of the system in a poor solvent, the PS ligands formed physical bonds between the NR ends, thereby leading to the formation of a polymer-like structure

\section{Homopolymerization}

To examine the effect of NR reactivity in copolymerization process, we studied the kinetics of the self-assembly of NRs with different length, L, in homopolymer structures. Figure 2a and $2 \mathrm{~b}$ show STEM images of the NR chains formed by $50 \mathrm{~nm}$ - and $80 \mathrm{~nm}$-long NRs, respectively. Each population of NRs underwent an end-to-end assembly in linear structures with a limited degree of branching. Similar to molecular polymerization, the growth of NR chains was characterized by the temporal change in their number-average degree of polymerization, $\overline{X_{n}}$, as

$$
\bar{X}_{\mathrm{n}}=\frac{\sum n_{\mathrm{x}} x}{\sum n_{\mathrm{x}}},
$$

where $\mathrm{x}$ is the number of NRs in the chain and $n_{\mathrm{x}}$ is the number of chains containing $x$ NRs. For the NRs with lengths of 50, 80 and $95 \mathrm{~nm}$, the value of $\overline{X_{\mathrm{n}}}$ increased linearly with the self-assembly time (Figure 2c), in a process analogous to reaction-controlled step-growth polymerization, ${ }^{[1,19]}$. With increasing NR length, the self-assembly rate decreased, that is, a longer time was needed to achieve a particular value of $\bar{X}_{\mathrm{n}}$. The rate constant $k$, calculated from the slope of the linear dependence of $\bar{X}_{\mathrm{n}} v s$. time $\left(k=\left(\mathrm{d} \bar{X}_{\mathrm{n}} / \mathrm{dt}\right) / 4[\mathrm{NR}]_{0}\right)$ was $3.72 \times 10^{5}$, $2.58 \times 10^{5}$ and $2.19 \times 10^{5}\left[\mathrm{M}^{-1} \mathrm{~s}^{-1}\right]$ for the NRs with the length of 50,80 and $95 \mathrm{~nm}$, respectively. 
The reduction in reactivity of longer NRs presumably originated from their reduced mobility (scaling as $1 / \mathrm{L}$ ), which led to a lower reaction frequency between NR ends. Moreover, longer NRs had a larger excluded volume $v_{\mathrm{e}}$ (scaling as $v_{\mathrm{e}} \sim \mathrm{L}^{2}$ ), ${ }^{[20]}$ which was not accessible to other NRs, as a result of steric repulsion (Figure 2d).

\section{Co-assembly of random copolymers}

Copolymerization of NRs with different reactivities was studied for the $50 \mathrm{~nm}$ - and $80 \mathrm{~nm}$ long NRs undergoing self-assembly with the difference in rate constants of $\sim 30 \%$. Later in the text, we refer to these NRs as to SNRs and LNRs, respectively. The individual solutions of SNRs and LNRs in DMF were mixed to achieve the ratio of number densities of SNRsto-LNRs of $0.8: 1.0$, with a total NR concentration of $0.18 \mathrm{nM}$. Following the addition of water to $C_{\mathrm{w}}=15 \mathrm{wt} \%$, the NRs with different lengths co-assembled in linear copolymer structures with a limited extent of branching. The STEM images of the copolymers were processed and analyzed using a Matlab program. Figure 3a shows a representative STEM image after Matlab processing, in which the SNRs and the LNRs are labelled with blue and red colors, respectively.

We analyzed the kinetics of the co-assembly of SNRs and LNRs by examining the variation of $\overline{X_{n}}$ with the self-assembly time. The value of $\overline{X_{n}}$ increased linearly with time (Figure $3 b$ ), consistent with numerical simulations of the reaction-controlled step-growth copolymerization (Supporting Information). The effective rate constant of copolymerization was determined as $k_{\mathrm{copol}}=\left(\mathrm{d} \bar{X}_{\mathrm{n}} / \mathrm{dt}\right) / 2\left([S]_{0}+[L]_{0}\right)$ and found to be $2.97 \times 10^{5} \mathrm{M}^{-1} \mathrm{~s}^{-1}$, where $[S]_{0}$ and $[L]_{0}$ are the initial concentrations of SNR and LNR ends, respectively. In the early stage of copolymerization, $k_{\mathrm{copol}}$ was expanded as

$$
k_{\mathrm{copol}}=k_{\mathrm{SS}} f^{2}+k_{\mathrm{LL}}(1-f)^{2}+2 k_{S L} f(1-f),
$$

where $f=[S]_{0} /\left([S]_{0}+[L]_{0}\right)$ is the fraction of SNRs at time $t=0$, and $k_{\mathrm{SS}}, k_{\mathrm{LL}}$ and $k_{\mathrm{SL}}$ are rate constants of the reaction between SNR chain-end and another SNR chain-end, a LNR chainend and another LNR chain-end, and a SNR chain-end and a LNR chain-end, respectively. Importantly, the value of $k_{\mathrm{SL}}$ of $2.89 \times 10^{5} \mathrm{M}^{-1} \mathrm{~s}^{-1}$ (calculated from Eq. 2) had an intermediate value between the rate constants of homopolymerization of SNRs and LNRs.

Next, we studied the temporal variation in the composition of copolymer chains, characterized as the fractions of SNRs $\left(\phi_{\mathrm{SNR}}\right)$ and LNRs $\left(\phi_{\mathrm{LNR}}\right)$ in the chains as

$$
\phi_{\mathrm{SNR}}=\frac{N_{\mathrm{SNR}}}{N_{\mathrm{SNR}}+N_{\mathrm{LNR}}} \text { and } \phi_{\mathrm{LNR}}=1-\phi_{\mathrm{SNR}} \text {, }
$$

where $N_{\mathrm{SNR}}$ and $N_{\mathrm{LNR}}$ are the numbers of SNRs and LNRs in the chains, respectively. Figure $3 \mathrm{c}$ shows the temporal variations in $\phi_{\mathrm{SNR}}$ and $\phi_{\mathrm{LNR}}$. After $1 \mathrm{~h}$, both NR types were co-assembled in the copolymer chains in close-to-equal amounts, although the initial ratio of number densities of SNRs-to-LNRs was 0.8:1.0. This effect was resulted from the partial compensation of the higher reactivity of SNRs by the higher concentration LNRs in the 
reaction system. With increasing self-assembly time, the fraction of SNRs in the chains decreased, due to their faster consumption, leading to the reduction of concentration of unreacted SNRs. For the opposite reason, the fraction of LNRs in the chains increased. After $8 \mathrm{~h}$, at conversion $p=0.875$ (calculated as $p=1-1 / \bar{X}_{\mathrm{n}}$ ), the fractions of SNRs and LNRs in the chains were 0.42 and 0.58 , respectively, and deviated by only 0.02 (or $3.5 \%$ ) from the values expected for complete conversion. The experimental results correlated with theoretical variation in copolymer composition, plotted on the same graph. The results shown in Figure $3 \mathrm{c}$ implied that at high conversion, the composition of copolymers will be represented, or will only slightly deviate from the initial composition of the co-monomer mixture, ${ }^{[22]}$ similar to molecular copolymerization.

The change in composition of the reaction mixture has important consequences: while we observed a linear growth of $\overline{X_{n}} v s$. time (Figure $3 \mathrm{~b}$ ), after a sufficiently long time this dependence is expected to become non-linear. The time interval required for the deviation from linearity to appear reduces with a larger difference between $k_{\mathrm{LL}}$ and $k_{\mathrm{SS}}$ (Figure S2a), that is, with increasing "asymmetry" of the system. However, even for $k_{\mathrm{LL}} / k_{\mathrm{SS}} \ll 1$, the nonlinear effects are predicted to be weak, if the concentration of LNRs is considerably lower than that of SNRs. In this case, the reaction is dominated by the SNRs, as they have a higher concentration and react faster (Figure S3).

Finally, the microstructure of the copolymer was characterized by using a microheterogeneity coefficient, $K$, as ${ }^{[23]}$

$$
K=\frac{P_{\mathrm{SNR}-\mathrm{LNR}}}{P_{\mathrm{SNR}-\mathrm{LNR}}+2 P_{\mathrm{SNR}-\mathrm{SNR}}}+\frac{P_{\mathrm{SNR}-\mathrm{LNR}}}{P_{\mathrm{SNR}-\mathrm{LNR}}+2 P_{\mathrm{LNR}-\mathrm{LNR}}},
$$

where $P_{\mathrm{SNR}-\mathrm{LNR}}, P_{\mathrm{SNR}-\mathrm{SNR}}$ and $P_{\mathrm{LNR}-\mathrm{LNR}}$ are the fractions of dyads of elementary monomer units SNR-LNR, SNR-SNR, and LNR-LNR, respectively. In molecular copolymerization, $K$ values of 0,1 , and 2 correspond to block, random and alternating copolymers, respectively. In our work, the values of $K=0.94$ and $K=1.01$ after the selfassembly times of 0.5 and $8 \mathrm{~h}$, respectively, showed a weak increase with time (Figure 3d), yet, the value of $K$ remained close to unity. We stress that in contrast with molecular copolymerization, in which an insignificant difference in co-monomer reactivities is generally assumed, in our experiments the SNR and LNR co-monomers had a $\sim 30 \%$ difference in the self-assembly rate constants. Nonetheless, after $8 \mathrm{~h}$ assembly, the difference in comonomer reactivity and the difference in their initial concentration, was not sufficient to induce block formation.

We extended the copolymerization approach to the co-assembly of co-monomers with different compositions, namely, palladium NRs with the mean length and diameter of 270 and $20 \mathrm{~nm}$, respectively, and gold NRs with the mean length and diameter of 183 and 19 $\mathrm{nm}$, respectively. The NRs were synthesized as described elsewhere. ${ }^{[24,25]}$ Following the synthesis, CTAB at the ends of the NRs was replaced with thiol-terminated PS molecules with the molecular weight $M_{\mathrm{n}}=50000 \mathrm{~g} \mathrm{~mol}^{-1}$. We first tested the homopolymerization of palladium NRs by adding water to the NR solution in DMF to $C_{\mathrm{w}}=15 \mathrm{wt} \%$. Figure $4 \mathrm{a}$ shows a representative STEM image of the palladium NRs assembled in the end-to-end manner, 
which confirmed the dominant regiospecific attachment of PS ligands to the NR ends. For the self-assembly time of $48 \mathrm{~h}$, a linear increase of $\bar{X}_{\mathrm{n}}$ with self-assembly time occurred with rate constant $k=3.2 \times 10^{2}\left[\mathrm{M}^{-1} \mathrm{~s}^{-1}\right]$. Due to the low rate of self-assembly, the chains reached $\overline{X_{\mathrm{n}}}$ of only 2.2. Furthermore, because of the large mass of palladium NRs, the chains with $x>10$ precipitated.

For the co-assembly of gold and palladium NRs, we mixed their individual solutions in DMF to achieve the ratio of number densities of gold-to-palladium NRs of 1:0.2. Following the addition of water to $C_{\mathrm{w}}=15 \mathrm{wt} \%$, the NRs co-assembled into copolymer structures. Figure $4 \mathrm{~b}$ shows an STEM image of copolymers formed after $30 \mathrm{~h}$ self-assembly. Palladium NRs were distinguished from gold NRs due to their rougher surface and a lower electron density. After $48 \mathrm{~h}$, the copolymer chains had a fraction of gold NRs of 0.72 and microheterogeneity coefficient $K=1.13$, which reflected a stronger asymmetry of the system than in the case of LNRs and SNRs. Yet, for the selected self-assembly time, the value of $K$ did not strongly deviate from unity, indicating the formation of a random copolymer.

\section{Co-assembly of block copolymers}

For the co-assembly of block copolymer structures from gold SNRs and LNRs, respectively, we used two synthetic strategies that are commonly utilized in the synthesis of molecular block copolymers. ${ }^{[19,26]}$ Figure 5a (left) shows a schematic of the two-prepolymer approach to NR block copolymers. We separately prepared homopolymer structures from SNRs and LNRs in the DMF/water mixture at $C_{\mathrm{w}}=15 \mathrm{wt} \%$, each at $\overline{X_{\mathrm{n}}} \approx 2$ by assembling them for 2 and 3 hours, respectively. Upon mixing the solutions of homopolymers, the chains of SNRs and of LNRs underwent copolymerization. In the one-prepolymer method (Figure 5a, right), after assembly of LNR homopolymer with $\overline{X_{n}}=5.5$ in a water/DMF mixture, we introduced individual SNRs. In a separate experiment, we assembled SNR chains and added individual LNRs (Figure S2).

The copolymerization of the LNRs and SNRs was monitored by imaging the self-assembled structures and by measuring their extinction spectra in the course of copolymerization. For the two-prepolymer method, a representative MATLAB-processed STEM image in Figure $5 \mathrm{~b}$ and $\mathrm{S} 5$ shows diblock and triblock copolymers, as well as individual NRs, homopolymers, and chains with short SNR-LNR fragments. Figure $5 \mathrm{c}$ shows the distribution of all the species in the system. A large ( $\sim 80 \%)$ population was represented by diblock and triblock copolymer structures. The value of microheterogeneity coefficient, $K=0.5$ indicated the formation of a large fraction of block copolymers.

For block copolymers prepared by one-prepolymer method, we analyzed the STEM images of the chains to examine the temporal change in $\overline{X_{\mathrm{n}}}$ of the LNR and SNR blocks (Figure 5d). While the value of $\overline{X_{n}}=5.5$ for the LNR blocks changed by less than $2 \%$, the value of $\bar{X}_{\mathrm{n}}$ of the SNR blocks showed the expected linear increase, due the dominant effect of the attachment of SNR monomers or oligomers to the ends of LNR blocks or to each other. This effect was expected for the ratio of concentrations of LNR chains and individual SNRs of 0.18:1.0, respectively (resulting from equal initial concentrations of individual SNRs and LNRs and from $\overline{X_{\mathrm{n}}}=5.5$ for the LNR prepolymer). 
The dominant growth of SNR blocks was also supported by the variation in extinction spectra of plasmonic chains (Figure 5e), which exhibited red shift of the $\lambda_{\text {LSPR }}$ corresponding to SNRs. ${ }^{[8,14]}$ With progressing co-assembly time, the $\lambda_{\text {LSPR }}$ corresponding to the chains of LNRs remained invariant at $1221 \mathrm{~nm}$, indicating that the formation of new LNR-LNR contacts was negligible. Concurrently, the $\lambda_{\text {LSPR }}$ of the SNRs red-shifted from 835 to $916 \mathrm{~nm}$, suggesting that individual SNRs co-assembled in an end-to-end manner either by forming chains of SNRs, or by forming SNRs blocks attached to the LNR blocks. While the association of LNR chains with SNRs or with chains of SNRs did not lead to a shift of $\lambda_{\text {LSPR }}$ of the LNR chains, a slight increase of the peak intensity was observed, presumably because the LSPR of the LNR chains was close to saturation. ${ }^{[14]}$ A similar change in extinction was observed for block copolymers produced by adding LNR monomers to a SNR prepolymer: the $\lambda_{\mathrm{LSPR}}$ of SNR chains remained constant, while the value of $\lambda_{\text {LSPR }}$ of the LNRs red-shifted (Figure S6).

In summary, our work provides "molecular" guidance for the generation of one-dimensional plasmonic heterostructures (plasmonic copolymers) that are formed by nanoparticles with distinct dimensions and compositions. These structures uniquely match the needs of single particle spectroscopy and are challenging to achieve by top-bottom approach. They provide an excellent model system for the fundamental studies of plasmonic properties (such as Fano plasmon modes) that are caused by the asymmetry of nanostructures with unequally sized building blocks. Furthermore, the ability to visualize self-assembled nanostructures allows for the analysis of different stages of copolymerization process, and in contrast to molecular polymerization, reveals the mechanism of copolymerization of "asymmetric" monomers.

\section{Experimental Section}

The self-assembled NR structures were imaged by STEM (Hitachi HD-2000) at $200 \mathrm{kV}$ and TEM (H-7000) at $100 \mathrm{kV}$. A dilute solution of the NRs or a dispersion of self-assembled NRs was cast onto a carbon-coated copper grid and the solvent was quickly removed with a Kimwipe.

Analysis of STEM images was performed using a MATLAB program. Each NR was approximated as an ellipsoid and the length of the NR was approximated as the major axis of the ellipsoid. The threshold between populations of SNRs and LNRs was set at $62.5 \mathrm{~nm}$, allowing the analysis of the fraction of LNR-LNR, SNR-SNR, SNR-LNR connectivity.

\section{Supplementary Material}

Refer to Web version on PubMed Central for supplementary material.

\section{Acknowledgments}

EK thanks NSERC Canada for financial support this work through BiopSys Strategic Network Grant. KL thanks the Ontario Ministry of Economic Development and Innovation for the Post-Doctoral Fellowship, 1000 Young Talent Plan of China funds, and the startup funds from Jilin University. M.R. acknowledges support from the National Science Foundation under grants CHE-0911588, DMR-0907515, DMR-1309892, DMR-1121107, and DMR-1122483, the National Institutes of Health under 1-P5-HL107168, 1-P01-HL108808-01A1 and the Cystic Fibrosis Foundation. The authors thank Dr. Sergei Panyukov for useful discussions. 


\section{References}

1. a) Wang Y, Wang Y, Breed DR, Manoharan VN, Feng L, Hollingsworth AD, Weck M, Pine DJ. Nature. 2012; 491:51-55. [PubMed: 23128225] b) Bharti B, Findenegg GH, Velev OD. Sci Rep. 2012; 2:Art. number: 1004A.c) Gröschel AH, Schacher FH, Schmalz H, Borisov OV, Zhulina EB, Walther A, Müller AHE. Nat Commun. 2012; 3:710. [PubMed: 22426231] d) Rupar PA, Chabanne L, Winnik MA, Manners I. Science. 2012; 337:559-562. [PubMed: 22859484] e) Cui H, Chen Z, Zhong S, Wooley KL, Pochan DJ. Science. 2007; 317:647-650. [PubMed: 17673657] f) Liu K, Nie Z, Zhao N, Li W, Rubinstein M, Kumacheva E. Science. 2010; 329:197-200. [PubMed: 20616274] g) Klinkova, Thérien-Aubin H, Choueiri RM, Rubinstein M, Kumacheva E. Proc Nat Acad Sci USA. 2013; 110:18775-18779. [PubMed: 24190993]

2. a) Keng PY, Kim BY, Shim IB, Sahoo R, Veneman PE, Armstrong NR, Yoo H, Pemberton JE, Bull MM, Griebel JJ, Ratcliff EL, Nebesny KG, Pyun J. ACS Nano. 2009; 3:3143-3157. [PubMed: 19799415] b) Wang L, Zhu Y, Xu L, Chen W, Kuang H, Liu L, Agarwal A, Xu C, Kotov NA. Angew Chem, Int Ed. 2010; 49:5472-5475.c) Zhang H, Liu Y, Yao D, Yang B. Chem Soc Rev. 2012; 41:6066-6088. [PubMed: 22641116] d) Liu K, Zhao N, Kumacheva E. Chem Soc Rev. 2011; 40:656-671. [PubMed: 21218236]

3. Slaughter LS, Willingham BA, Chang WS, Chester MH, Ogden N, Link S. Nano Lett. 2012; 12:3967-3972. [PubMed: 22738257]

4. Ozbay E. Science. 2006; 311:189-193. [PubMed: 16410515]

5. Maier SA, Kik PG, Atwater HA, Meltzer S, Harel E, Koel BE, Requicha AAG. Nat Mater. 2003; 2:229-232. [PubMed: 12690394]

6. Kosmeier S, De Luca AC, Zolotovskaya S, Di Falco A, Dholakia K, Mazilu M. Sci Rep. 2013; 3:1808. [PubMed: 23657743]

7. Chen G, Wang Y, Yang M, Xu J, Goh SJ, Pan M, Chen H. J Am Chem Soc. 2010; 132:3644-3645. [PubMed: 20196540]

8. a) Lukach A, Liu K, Therien-Aubin H, Kumacheva E. J Am Chem Soc. 2012; 134:18853-18859. [PubMed: 23078101] b) Barrow SJ, Funston AM, Gómez DE, Davis TJ, Mulvaney P. Nano Lett. 2011; 11:4180-4187. [PubMed: 21879717] c) Liu K, Ahmed A, Chung S, Sugikawa K, Wu G, Nie Z, Gordon R, Kumacheva E. ACS Nano. 2013; 7:5901-5910. [PubMed: 23786318]

9. Huang CP, Yin XG, Kong LB, Zhu YY. J Phys Chem C. 2010; 114:21123-21131.

10. a) Lombardi A, Grzelczak MP, Crut A, Maioli P, Pastoriza-Santos I, Liz-Marzán LM, Del Fatti N, Vallée F. ACS Nano. 2013; 7:2522-2531. [PubMed: 23418719] b) Sheikholeslami S, Jun YW, Jain PK, Alivisatos AP. Nano Lett. 2010; 10:2655-2660. [PubMed: 20536212]

11. a) Pyun J. Angew Chem, Int Ed. 2012; 51:12408-12409.b) Zhang H, Wang D. Angew Chem, Int Ed. 2008; 47:3984-3987.c) Yang M, Chen G, Zhao Y, Silber G, Wang Y, Xing S, Han Y, Chen H. Phys Chem Chem Phys. 2010; 12:11850-11860. [PubMed: 20661507] d) Wang, Chen L, Shen X, Zhu L, He J, Chen H. Angew Chem, Int Ed. 2012; 51:8021-8025.e) Xia H, Su G, Wang D. Angew Chem, Int Ed. 2013; 52:3726-3730.f) Nie Z, Fava D, Kumacheva E, Zou S, Walker GC, Rubinstein M. Nat Mater. 2007; 6:609-614. [PubMed: 17618291]

12. a) Nie Z, Petukhova A, Kumacheva E. Nat Nanotechnol. 2010; 5:15-25. [PubMed: 20032986] b) Choi CL, Alivisatos AP. Annu Rev Phys Chem. 2010; 61:369-389. [PubMed: 20055683]

13. a) Zhao Y, Xu L, Liz-Marzán LM, Kuang H, Ma W, Asenjo-Garcia A, García de Abajo FJ, Kotov NA, Wang L, Xu C. J Phys Chem Lett. 2013; 4:641-647.b) Gröschel AH, Walther A, Löbling TI, Schacher FH, Schmalz H, Müller AHE. Nature. 2013; 503:247-251. [PubMed: 24185010]

14. Yang ZJ, Zhang ZS, Zhang W, Hao ZH, Wang QQ. Appl Phys Lett. 2010; 96:131113.

15. Slaughter L, Chang WS, Link S. J Phys Chem Lett. 2011; 2:2015-2023.

16. Nikoobakht B, El-Sayed MA. Chem Mater. 2003; 15:1957-1962.

17. Ni W, Kou X, Yang Z, Wang J. ACS Nano. 2008; 2:677-686. [PubMed: 19206598]

18. Link S, Mohamed MB, El-Sayed MA. J Phys Chem B. 1999; 103:3073-3077.

19. Gritsenko TM. J Macromol Sci-Chem. 1975; 9:357-371.

20. Rubinstein, M.; Colby, RH. Polymer Physics. Oxford University Press; Oxford: 2003.

21 . The number of NRs overlapping in length between the two populations was $<10 \%$. 
22. Odian, G. Principles of Polymerization. 4. Wiley; New York: 2004.

23. Kuchanov S, Slot H, Stroeks A. Prog Polym Sci. 2004; 29:563-633.

24. Chen YH, Hung HH, Huang MH. J Am Chem Soc. 2009; 131:9114-9121. [PubMed: 19507854]

25. Busbee BD, Obare SO, Murphy CJ. Adv Mater. 2003; 15:414-416.

26. Hadjichristidis, N. Block Copolymers. Wiley; New York: 2002. 

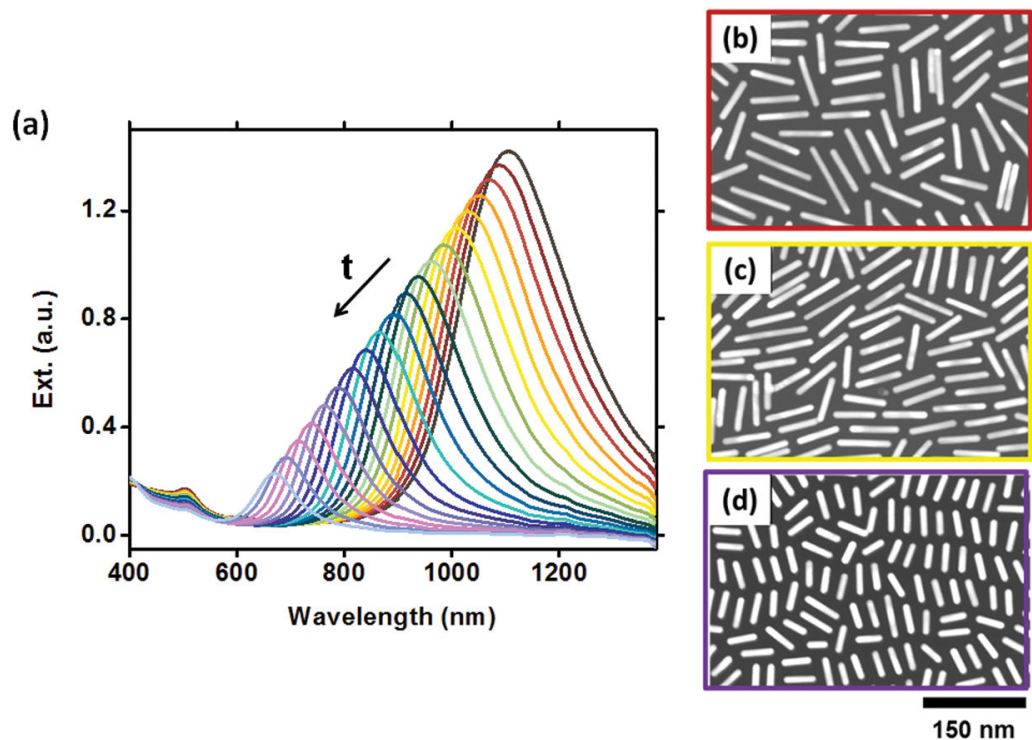

Figure 1.

Tuning the length of gold NRs. (a) Temporal variation in extinction spectra of the NRs in the course of the etching process. Starting from the $99 \mathrm{~nm}$-long NRs at beginning of etching process at $\mathrm{t}=0$ (black curve), the spectra were acquired every $5 \mathrm{~min}$. (b-d) STEM images of the NRs with average lengths of (b) $95 \mathrm{~nm}$, (c) $80 \mathrm{~nm}$, and (d) $50 \mathrm{~nm}$. The mean diameter of the NRs was constant at $12 \mathrm{~nm}$. The frame color around the STEM images matches the color of the corresponding extinction spectrum. 
(a)

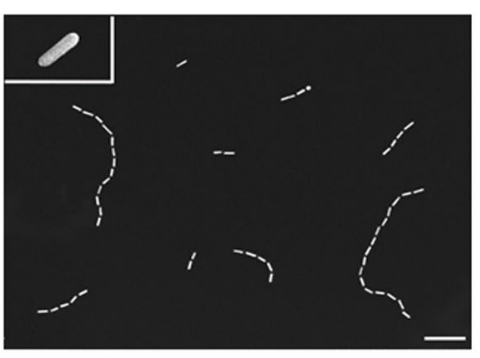

(b)

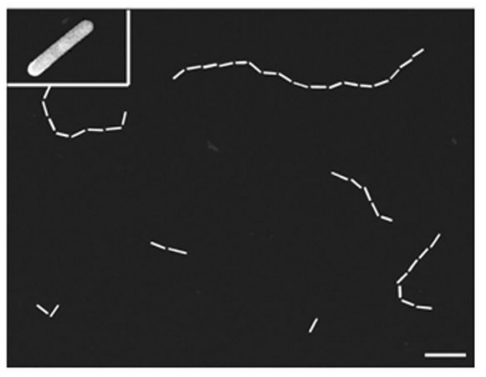

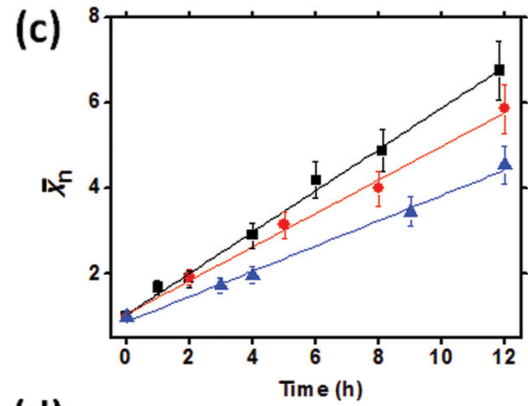

(d)

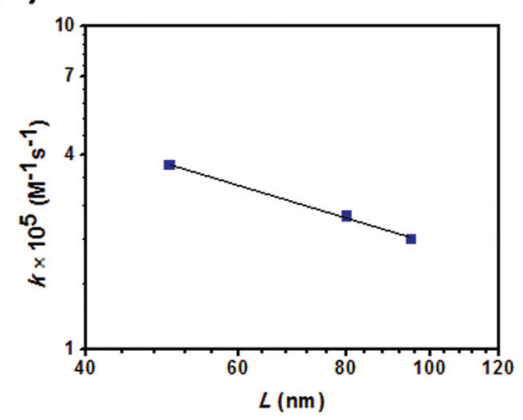

Figure 2.

Homopolymerization of NRs with different lengths. (a-b) STEM images of the NR chains formed after 4 h-long self-assembly time from (a) $50 \mathrm{~nm}$ - and (b) $80 \mathrm{~nm}$-long NRs. The insets show high magnification STEM images of the corresponding individual NRs. $C_{\mathrm{W}}=15 \mathrm{wt} \%$. Scale bars are $200 \mathrm{~nm}$. (c) Variation in the average degree of polymerization, $\overline{X_{\mathrm{n}}}$, of the NR chains with self-assembly time, for $50 \mathrm{~nm}$-long (black squares), $80 \mathrm{~nm}$-long (red circles), and $95 \mathrm{~nm}$-long (blue triangles) NRs at $[\mathrm{M}]_{0}=0.1 \mathrm{nM} .^{[21]}$. The lines are linear fits. (d) Variation in the rate constant of self-assembly of the NRs with different mean lengths. The slope is -0.81 . 
(a)

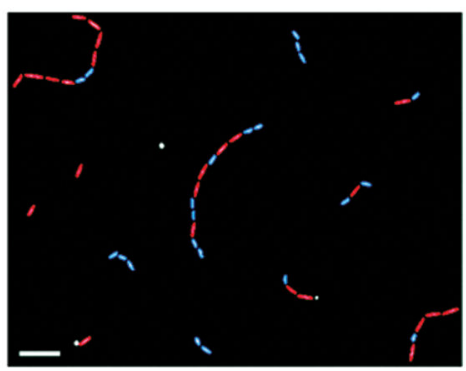

(c)

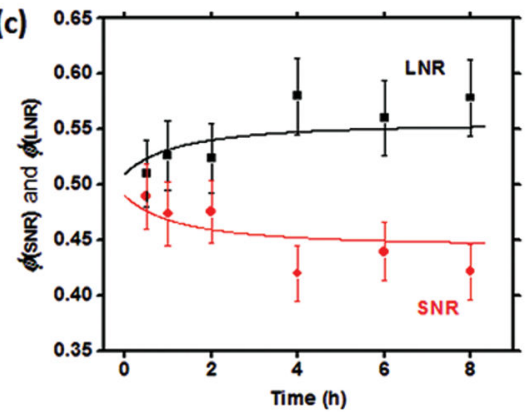

(b)

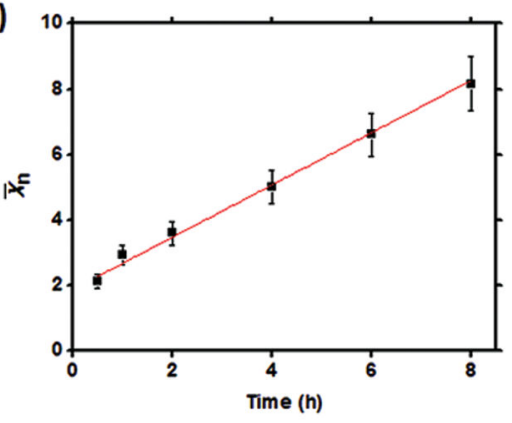

(d)

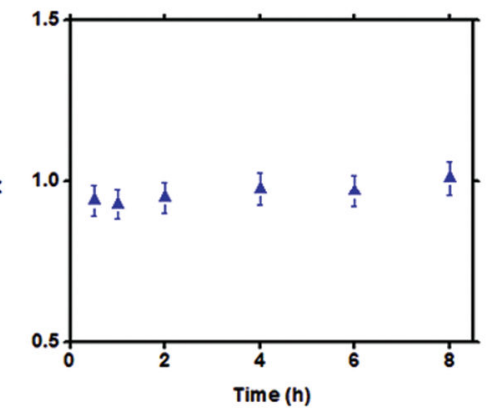

Figure 3.

Co-assembly of gold NRs with different lengths. (a) Matlab program-processed STEM image of chains formed by SNRs (blue color) and LNRs (red color). The scale bar is $200 \mathrm{~nm}$. (b) Variation in the average degree of polymerization of the chains formed from SNRs and LNRs, plotted as a function of self-assembly time. (c) Temporal variation of the fractions of SNRs and LNRs in the chains. (d) Variation in the microheterogeniety coefficient with self-assembly time. The mean length of the SNRs and LNRs was 50 and $80 \mathrm{~nm}$, respectively. Each experimental point was calculated based on the analysis of 250-550 chains (a total of 600-2200 NRs). 

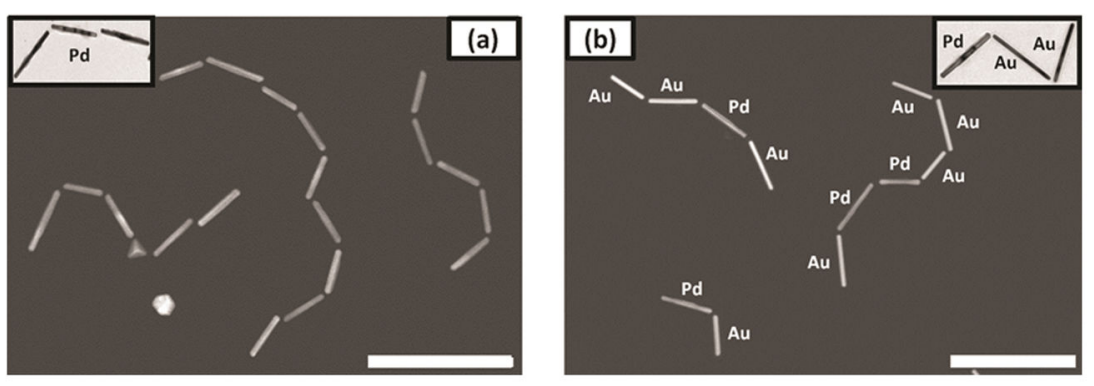

Figure 4.

Copolymerization of palladium and gold NRs. (a-b) STEM images of (a) Homopolymers of palladium NRs, and (b) Copolymers of palladium and gold NRs with initial concentrations of 8.6 and $43 \mathrm{nM}$, respectively. Insets: high magnification transmission electron microscopy (TEM) image of fragments of (a) a palladium (Pd) NR chain and (b) a Pd-gold (Au) chain. $C_{\mathrm{w}}=15 \mathrm{wt} \%$. The self-assembly time was $48 \mathrm{~h}$. Scale bars are $500 \mathrm{~nm}$. 
(a)

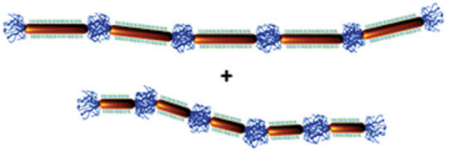

(b)

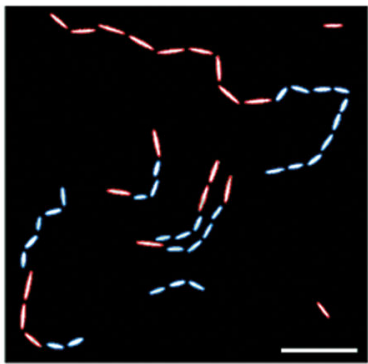

(c)

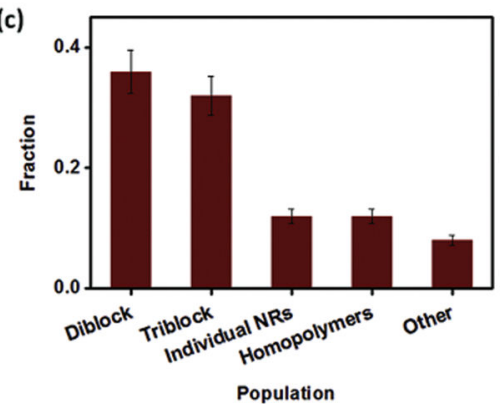

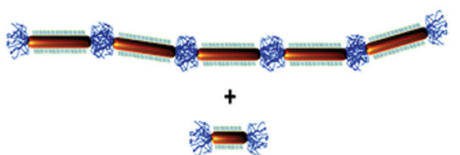

(d)

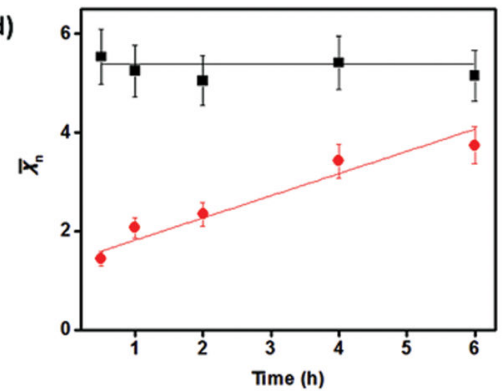

(e)

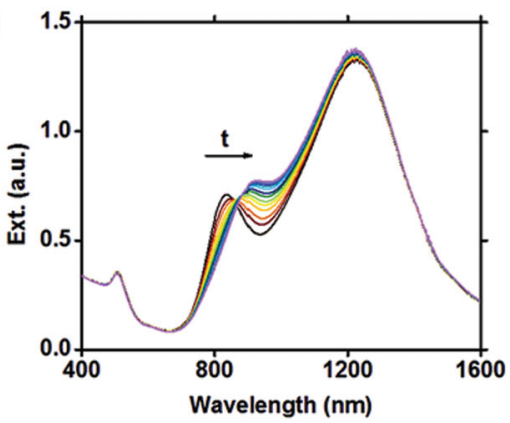

Figure 5.

Formation of block copolymer from SNRs and LNRs. (a) Schematic illustration of two-prepolymer (left) and one-prepolymer (right) methods for step-growth copolymerization of block copolymers. (b) MATLAB-processed STEM image of the chains formed by the two-prepolymer method. Scale bar is $200 \mathrm{~nm}$. (c) Distribution of species following NR co-assembly via a twoprepolymer method. "Other" refers to multiblock and branched chains. (d) Temporal variation in X̄n of blocks formed by SNRs (red circles) and LNRs (black squares) in copolymers assembled by a one-prepolymer method. (e) Extinction spectra of the SNRs and LNRs undergoing copolymerization via a one-prepolymer approach. 